\title{
DOENÇA DO REFLUXO GASTROESOFÁGICO: revisão ampliada
}

\author{
Ary NASI ${ }^{1}$, Joaquim Prado P. de MORAES-FILHO ${ }^{2}$ e Ivan CECCONELLO ${ }^{1}$
}

RESUMO - Racional - A doença do refluxo gastroesofágico, considerada uma das afecções digestivas de maior prevalência nos países ocidentais, tem sido bastante estudada. Merece destaque o grande número de publicações existentes na literatura médica sobre o tema. Contudo, há controvérsia em relação a vários aspectos relevantes da afecção. Objetivos - Salientar tópicos importantes da doença e apresentar revisão de literatura do tema. Foram revistos os seguintes tópicos: definição, prevalência, manifestações clínicas, diagnóstico, indicações e limitações dos principais métodos diagnósticos e tratamento clínico, cirúrgico e endoscópico. Material e métodos - Pesquisou-se, via Internet: artigos de revisão, consensos, "guidelines " e revisões sistemáticas de literatura com meta-análise, publicados nos últimos 5 anos (2000 a 2005), nas seguintes bases de dados: Central de Registro de Estudos Controlados da Cochrane Library e na PubMed. Dentre muitos trabalhos encontrados, foram selecionados nove revisões sistemáticas de literatura com meta-análise, cinco revisões de literatura com critérios de inclusão bem definidos, cinco "guidelines" e quatro publicações de consensos, que abordassem os tópicos propostos para a revisão. Vale salientar que, como se observa nas referências bibliográficas, não foram consideradas apenas as publicações encontradas na referida pesquisa; outras publicações relevantes (algumas anteriores ao período de tempo analisado) foram consideradas na redação do presente texto. Conclusões - Dentre as 12 conclusões apresentadas, destacam-se duas: 1. a impedanciometria esofágica tem contribuído substancialmente para o melhor conhecimento da doença do refluxo gastroesofágico e se desponta, quando associada à pHmetria (impedanciopHmetria esofágica), como o novo padrão-ouro para o diagnóstico do refluxo gastroesofágico, e 2. dentre os sintomas da afecção há os que podem ser considerados ácido-dependentes que costumam responder bem ao tratamento com inibidores de bomba de prótons. Contudo, há também sintomas que são mais dependentes da presença física do refluxo do que da sua acidez. Esses, que não dependem tanto do teor ácido do refluxo, não são bem controlados pelo uso de anti-secretores.

DESCRITORES - Refluxo gastroesofágico. Esofagite. Transtornos da motilidade esofágica.

\section{INTRODUÇÃO}

A doença do refluxo gastroesofágico (DRGE), considerada uma das afecções digestivas de maior prevalência nos paises ocidentais ${ }^{(22)}$, tem sido bastante estudada. Merece destaque o grande número de publicações existentes na literatura médica sobre a mesma. Contudo, há controvérsia em relação a vários aspectos relevantes da afecção.

O objetivo do presente estudo foi salientar tópicos importantes da doença e apresentar revisão de literatura do tema. Foram revistos os seguintes tópicos: definição, prevalência, manifestações clínicas, diagnóstico, indicações e limitações dos principais métodos diagnósticos e tratamento clínico, cirúrgico e endoscópico.
Estratégia de pesquisa e critérios de seleção de trabalhos

Pesquisou-se, via Internet, artigos de revisão, consensos, "guidelines" e revisões sistemáticas de literatura com meta-análise, publicados nos últimos 5 anos (2000 a 2005), nas seguintes bases de dados: Central de Registro de Estudos Controlados da Cochrane Library e na PubMed. Dentre muitos trabalhos encontrados, foram selecionados nove revisões sistemáticas de literatura com meta-análise, cinco revisões de literatura com critérios de inclusão bem definidos, cinco "guidelines" e quatro publicações de consensos, que abordassem os tópicos propostos para a revisão. Vale salientar que, como se observa nas referências bibliográficas, não foram consideradas apenas as publicações encontradas

Disciplinas de ${ }^{1}$ Cirurgia do Aparelho Digestivo e Coloproctologia e ${ }^{2}$ Gastroenterologia Clínica da Faculdade de Medicina da Universidade de São Paulo. Endereço para correspondência: Dr. Ary Nasi - Rua Oscar Freire, 1702 - apt.88 - 05409-011 - São Paulo, SP. E-mail: ary.nasi@fleury.com.br 
na referida pesquisa; outras publicações relevantes (algumas anteriores ao período de tempo analisado) foram consideradas na redação do presente.

\section{Definição}

Definição bastante interessante da afecção é a sugerida pelo I Consenso Brasileiro sobre Doença do Refluxo Gastroesofágico ${ }^{(22)}$ : é a afecção crônica decorrente do fluxo retrógrado de parte do conteúdo gastroduodenal para o esôfago e/ou órgãos adjacentes, acarretando variável espectro de sintomas (esofágicos ou extra-esofágicos), associados ou não a lesões teciduais. Convém destacar três aspectos importantes na definição apresentada:

- admite-se a participação de componentes do refluxo duodenogástrico na fisiopatogenia da afecção. Em função disso, propõe-se o termo refluxo de conteúdo gastroduodenal ("não-ácido") e não apenas de conteúdo gástrico (ácido);

- admite-se a existência de sintomas esofágicos e extraesofágicos;

- destaca-se que os sintomas podem ou não ser acompanhados por lesões teciduais esofágicas diagnosticadas pelo exame endoscópico.

\section{Prevalência}

A DRGE é afecção de grande importância médico-social pela elevada e crescente incidência e por determinar sintomas de intensidade variável, que se manifestam por tempo prolongado, podendo prejudicar consideravelmente a qualidade de vida do paciente. Tem prevalência estimada de $20 \%$ na população adulta dos $\mathrm{EUA}^{(34)}$ e taxas similares na Europa.

No Brasil, estudo populacional empreendido em 22 metrópoles, entrevistando-se amostra populacional de 13.959 indivíduos $^{(23)}$, observou-se que $4,6 \%$ das pessoas entrevistadas apresentavam pirose uma vez por semana e que $7,3 \%$ apresentavam tal queixa duas ou mais vezes por semana. Em função desses dados, estima-se que a prevalência da DRGE, em nosso meio, seja ao redor de $12 \%$

\section{Manifestações clínicas e diagnóstico}

O primeiro passo para o diagnóstico adequado da DRGE é o conhecimento do conceito atual da afecção e das suas várias formas de apresentação clínica. As manifestações clínicas consideradas típicas da DRGE são pirose e regurgitação. Apesar desses sintomas sugerirem a presença da afecção, vale salientar que outras doenças (como úlcera péptica, gastrites e eventualmente neoplasias) podem cursar com um deles. Contudo, quando tais queixas co-existem, a possibilidade do paciente ter DRGE é superior a $90 \%$.

Mais recentemente, reconheceu-se que outras manifestações clínicas podem ser decorrentes de refluxo gastroesofágico (RGE). Considera-se que dor torácica não-coronariana, sensação de globus faríngeo, manifestações extra-esofágicas respiratórias (fundamentalmente tosse e asma brônquica) e otorrinolaringológicas (basicamente disfonia e pigarro) possam também ser decorrentes de RGE.

Lembrar que a ausência de manifestações típicas do refluxo (pirose e regurgitação) não exclui o diagnóstico da DRGE.
Pacientes com manifestações atípicas freqüentemente não apresentam sintomas típicos do refluxo ${ }^{(25)}$.

São cabíveis dois tipos de abordagem inicial em pacientes com DRGE: tratamento empírico (teste terapêutico) e tratamento baseado na confirmação diagnóstica da afecção por exames subsidiários. Na decisão sobre a abordagem a ser adotada, é importante considerar a idade e a presença ou não de manifestações de alarme. Recomenda-se confirmação diagnóstica em pacientes com idade superior a 40 anos, na presença de manifestações de alarme, tais como disfagia, odinofagia, anemia, hemorragia digestiva e emagrecimento, nos indivíduos com história familiar de câncer e naqueles com queixas de náuseas e vômitos e/ou sintomas de grande intensidade ou de ocorrência noturna ${ }^{(10,11,24)}$.

Quando, no entanto, está-se frente a manifestações típicas em pacientes com menos de 40 anos de idade, sem manifestações de alarme, pode-se considerar a instituição do teste terapêutico. Nesses casos é prescrito um inibidor da bomba protônica (IBP) em dose plena diária por 4 semanas, como conduta inicial. A resposta satisfatória, com remissão dos sintomas, sugere o diagnóstico da DRGE.

Entretanto, a respeito do teste terapêutico, é necessária cautela porque estudo baseado em revisão sistemática de literatura ${ }^{(26)}$ conclui que: "o teste terapêutico com inibidores de bomba protônica, em pacientes com suspeita clínica de DRGE, não estabelece o diagnóstico confiável da afecção". Justificando tal afirmação, destaca-se que o RGE pode desencadear sintomas decorrentes da acidez do material refluído (ex: pirose retroesternal). Contudo, pode também determinar sintomas que são mais dependentes da presença física do refluxo que da sua acidez propriamente dita (ex: regurgitação e muitas das manifestações extra-esofágicas). Os sintomas decorrentes da acidez do refluxo costumam melhorar com o uso de medicamentos anti-secretores (os mais usados são os IBP). Porém, os sintomas decorrentes da presença física do refluxo (independentes da sua acidez) não são adequadamente controlados por tais drogas. Portanto, havendo melhora da queixa clínica durante o teste terapêutico com IBP, sugere-se fortemente a participação do refluxo gastroesofágico. Caso contrário, não se pode descartar o diagnóstico da DRGE pois há duas situações que podem justificar o insucesso do teste: 1 . os sintomas apresentados pelo paciente podem ser decorrentes de refluxo "não-ácido" que não é adequadamente tratado pelos medicamentos anti-secretores; 2. os sintomas podem ser decorrentes de refluxo ácido não adequadamente bloqueado pelo IPB em uso.

\section{Indicações e limitações dos principais métodos diagnósticos}

\section{Endoscopia digestiva alta \\ Indicaçoes:}

- avalia a presença e o grau de esofagite (é considerado o melhor método para tal diagnóstico). Lembrar que existe esofagite endoscópica em cerca de $50 \%$ dos pacientes com DRGE, com predomínio de manifestações típicas (pirose e regurgitação) e em apenas $15 \%$ a $20 \%$ daqueles com predomínio de manifestações atípicas. Portanto, a ausência de esofagite ao estudo endoscópico não exclui o diagnóstico da $\operatorname{DRGE}^{(8)}$. 
- caracteriza a presença de complicações da DRGE (esôfago de Barrett, estenose e ulcerações esofágicas);

- evidencia afecções associadas (hérnia hiatal, úlceras pépticas gastroduodenais, neoplasias, etc.)

Limitaçōes:

Não avalia o RGE (apenas eventuais conseqüências do mesmo).

pHmetria esofágica prolongada:

Avalia a presença e a intensidade do refluxo ácido gastroesofágico; caracteriza o padrão de refluxo (ortostático, supino ou combinado) e relaciona a queixa clínica com o refluxo ácido gastroesofágico.

Indicaçōes:

- identificação dos portadores da DRGE sem esofagite;

- caracterização do padrão de refluxo;

- estudo da participação do refluxo ácido nas manifestações atípicas do refluxo (nessa situação, recomenda-se pHmetria com dois ou mais canais de registro: um posicionado no esôfago distal, para caracterizar RGE, outro posicionado no esfíncter superior do esôfago ou logo acima dele, para caracterização de refluxo supraesofágico ou também chamado de laringofaríngeo);

- estudo da recidiva dos sintomas no pós-operatório;

- avaliação da eficácia do tratamento clínico (nessa situação, também é recomendada a pHmetria com dois ou mais canais de registro: um posicionado no estômago, para avaliar a eficiência da droga em uso na neutralização da acidez gástrica, outro no esôfago inferior para caracterizar RGE.

\section{Limitaçóes:}

Não avalia a presença de esofagite e nem de complicações da mesma e nem a ocorrência de refluxo "não-ácido" (freqüentemente denominado, erroneamente, refluxo alcalino).

A endoscopia digestiva alta e a pHmetria esofágica prolongada são os dois métodos diretamente relacionados com o diagnóstico da DRGE. O primeiro identifica o sub-grupo da doença que cursa com esofagite; o segundo caracteriza o grupo com RGE patológico sem esofagite. A endoscopia propicia ainda a realização de coleta de material de biopsia para estudo histológico. Tal procedimento é de fundamental importância no estudo das complicações da doença. A seguir, são destacadas as indicações e limitações de outros métodos diagnósticos que, apesar de não se prestarem para o diagnóstico da DRGE em si, têm utilidade na prática clínica.

Estudo radiológico contrastado do esôfago

\section{Indicações:}

- propicia boa avaliação morfológica do órgão (forma do esôfago e características do trânsito da substância de contraste);

- pode evidenciar complicações da DRGE (estenoses e ulcerações) e condições favorecedoras do refluxo (hérnia hiatal e ângulo esôfago-gástrico anormal).

\section{Limitaçóes:}

Não identifica esofagite (apenas eventuais complicações desta) e não caracteriza adequadamente o RGE.

\section{Manometria esofágica}

Avalia o tônus pressórico dos esfíncteres do esôfago e a atividade motora do corpo esofágico ${ }^{(2)}$.

\section{Indicações:}

- fornece bom parâmetro preditivo de evolução da doença (casos com hipotonia muito acentuada do esfíncter inferior do esôfago freqüentemente necessitam de tratamento medicamentoso continuado, para controle adequado dos sintomas. Nesses casos, cogita-se mais precocemente a opção do tratamento cirúrgico do refluxo);

- diagnóstico de distúrbios motores específicos do esôfago, que podem alterar a orientação terapêutica (presença de acalásia, aperistalse e hipocontratilidades muito acentuadas);

- fornece bom parâmetro da atividade motora no pré-operatório de cirurgia anti-refluxo. Na presença de complicação disfágica, no pós-operatório de fundoplicatura, a avaliação manométrica do esôfago tem mais chance de poder auxiliar no esclarecimento da causa da disfagia nos pacientes submetidos a manometria pré-operatória (a manometria prévia fornece bom parâmetro referencial, aumentando a chance de esclarecimento diagnóstico da causa da disfagia);

- identificação da localização dos esfíncteres do esôfago, para posicionamento adequado dos sensores de pHmetria (para esse fim, não é necessário estudo manométrico completo do esôfago; realiza-se estudo sumário, apenas para identificação dos esfíncteres).

\section{Limitaçóes:}

Não avalia a real capacidade que o esôfago tem de transportar as substâncias ingeridas. Há alterações manométricas (pressóricas) que comprometem bastante a função esofágica daquele transporte (ex. acalásia, acometimento importante do esôfago por colagenoses e espasmo difuso do esôfago). Por outro lado, há alterações que não comprometem substancialmente tal capacidade de transporte ("nutcraker esophagus", hipocontratilidades pouco acentuadas do corpo esofágico, etc.) $)^{(38)}$.

\section{Impedanciometria esofágica}

A impedanciometria intraluminar esofágica é um novo método que possibilita o acompanhamento do movimento anterógrado (transporte das substâncias ingeridas) e do movimento retrógrado (RGE) do conteúdo intraluminar esofágico. Contudo, ressalta-se que a disponibilidade do método na prática clínica assistencial, ainda é limitada.

Quando associada à manometria esofágica, permite avaliar o transporte das substâncias ingeridas simultaneamente ao estudo da atividade pressórica intraluminar, que o promove. Com isso, pode-se relacionar a atividade pressórica intra-esofágica (avaliada pela manometria) com a real capacidade de transporte do esôfago (avaliada pela impedanciometria).

Associando-se a impedanciometria com a pHmetria (impedanciopHmetria esofágica), pode-se avaliar o movimento retrógrado do material refluído, caracterizar sua natureza física (líquido, gasoso ou misto) e química (ácido, não-ácido e levemente ácido). Com isso, pode-se verificar se ocorre refluxo, se este é líquido, gasoso ou misto e se é ácido ou não-ácido ${ }^{(30,32)}$.

Estudos recentes, em adultos e crianças, sugerem que a impedanciopHmetria tem potencial para ser o novo padrão-ouro para o diagnóstico da DRGE. TUTUIAN e CASTELL ${ }^{(37)}$, em recente revisão sobre o tema, afirmam que a presença e a ascensão proximal dos episódios de refluxo não-ácido, que ocorrem predominantemente 
no período pós-prandial (quando a acidez gástrica pode estar tamponada pelos alimentos ingeridos) e durante o tratamento com drogas anti-secretoras, podem agora ser adequadamente analisadas. A possibilidade de estudo da relação existente entre as manifestações sintomáticas do paciente e a presença de refluxo não-ácido pode ser de grande valor, sobretudo na avaliação dos sintomas em pacientes na vigência de drogas anti-secretoras.

A associação de $\mathrm{pH}$ e impedância também tem se mostrado útil no auxílio à indicação cirúrgica de alguns casos. TORQUATI et al. ${ }^{(3)}$, avaliando 13 pacientes com sintomas persistentes apesar do uso de IBP em dose dupla, observaram que 7 apresentavam sintomas relacionados com o refluxo: 1 deles com refluxo ácido e 6 com refluxo não-ácido. Isto significa que a maioria (6/7) dos pacientes apresentava sintomas persistentes devido a refluxo não-ácido, ou seja, não detectados pela $\mathrm{pHmetria}$ convencional. Dez pacientes foram submetidos a fundoplicatura e reavaliados, notando-se desaparecimento de sintomas e de refluxo. Isto indica que, diferentemente do tratamento com anti-secretores, o tratamento cirúrgico pode eliminar também o refluxo não-ácido.

\section{TRATAMENTO CLÍNICO}

Visa o alívio dos sintomas, a cicatrização das lesões e a prevenção de recidivas e complicações. Do ponto de vista prático, objetiva-se reduzir o potencial agressivo do conteúdo gástrico, minimizando a agressão representada pelo ácido clorídrico do suco gástrico ${ }^{(10)}$.

Pode-se classificar a abordagem terapêutica em medidas comportamentais e farmacológicas, que deverão ser implementadas simultaneamente.

\section{Medidas comportamentais}

As medidas comportamentais acham-se apresentadas no Quadro 1.

QUADRO 1 - Medidas comportamentais no tratamento da DRGE ${ }^{(22)}$

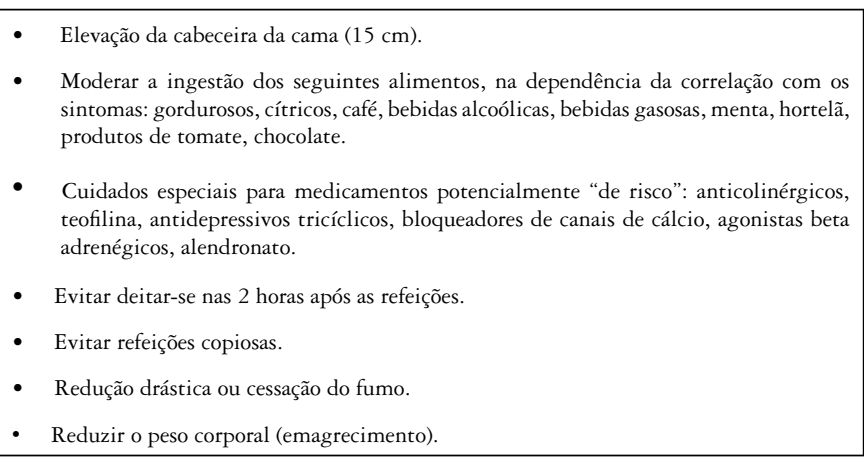

Apesar de não haver estudos sustentados por critérios de Medicina Baseada em Evidências em favor da utilização das medidas comportamentais, a experiência clínica tem sugerido fortemente a sua utilização (recomendação nível C).

\section{Medidas farmacológicas}

Os IBP aliviam os sintomas do refluxo e cicatrizam a esofagite mais rapidamente que os antagonistas $\mathrm{H}_{2}\left(\mathrm{AH}_{2}\right)^{(6,19)}$. As doses padrão utilizadas acham-se apresentadas no Quadro 2.
QUADRO 2 - Inibidores da bomba protônica e doses plenas diárias ${ }^{(22)}$

\begin{tabular}{|lc|}
\hline IBP & Dose plena diária \\
Omeprazol & $40 \mathrm{mg}$ \\
Lansoprazol & $30 \mathrm{mg}$ \\
Pantoprazol & $40 \mathrm{mg}$ \\
Rabeprazol & $20 \mathrm{mg}$ \\
Esomeprazol & $40 \mathrm{mg}$ \\
\hline
\end{tabular}

Contudo, apesar da eficiência dos IBP, como já destacado, existem casos que não têm boa resposta à droga. Há tendência em tratar os pacientes com diagnóstico de esofagite com IBP, em dose plena, por 6 a 12 semanas. Alguns autores ${ }^{(3,27)}$ propõem administração de dose dobrada (o dobro da dose plena) para as esofagites de maior gravidade (graus III e IV de Savary-Miller ou C e D de Los Angeles).

Estudo baseado em revisão sistemática de literatura ${ }^{(39)}$, avaliando a eficiência de IBP e $\mathrm{AH}_{2}$ em adultos com sintomas típicos da DRGE sem esofagite ao estudo endoscópico, conclui que: "os agentes antisecretores são eficientes no controle da queixa clínica desses pacientes e que os IBP apresentam resultados melhores que os $\mathrm{AH}_{2}$ ”.

Vários novos IBP (lansoprazol, rabeprazol e pantoprazol) têm eficiência semelhante ao omeprazol no controle da pirose, na taxa de cicatrização de esofagite e no controle da recidiva. Estudo baseado em revisão sistemática de literatura com meta-análise ${ }^{(4)}$, avaliando a eficiência dos novos IBP em comparação com omeprazol, ranitidina e placebo, na cicatrização e melhora dos sintomas decorrentes de DRGE, conclui que: "os novos IBP têm eficácia similar ao omeprazol em termos de controle da pirose, taxas de cicatrização e de recidiva dos sintomas. Todos os IBP foram melhores que a ranitidina e o placebo na cicatrização da esofagite".

A história natural da DRGE ainda não é bem compreendida. De fato, embora os sintomas sejam crônicos e muitas vezes recurrentes, usualmente a esofagite não progride com o passar do tempo. Assim, apenas pequena proporção (menos de 15\% dos pacientes sem esofagite ou com esofagite leve) progride para graus mais avançados da doença ${ }^{(20)}$. Vale salientar que cerca de $80 \%$ dos pacientes com DRGE apresentam recidiva dos sintomas nos 6 primeiros meses de interrupção do tratamento medicamentoso ${ }^{(21,29)}$. Nos casos em que é requerido o tratamento de manutenção, este deve ser feito utilizando-se IBP, empregandose a dose mínima eficaz para controle dos sintomas.

Outro estudo baseado em revisão sistemática de literatura ${ }^{(13)}$, avaliando a eficiência de IBP, $\mathrm{AH}_{2}$, procinéticos, sucralfate e placebo em adultos com DRGE (com e sem esofagite) conclui que os IBP são mais eficientes que as demais drogas. Contudo, apresentam maior ocorrência de efeitos colaterais que os placebos e maior ocorrência de cefaléia que a observada com os $\mathrm{AH}_{2}$. Os $\mathrm{AH}_{2}$ previnem recurrência de sintomas melhor que os placebos, justificando seu uso em pacientes com intolerância aos IBP. Tanto os procinéticos, quanto o sucralfato mostram benefícios em relação ao placebo".

O grande problema com o tratamento clínico da DRGE não é controlar os sintomas, mas sim manter os pacientes assintomáticos a longo prazo. Frente ao paciente que necessita de tratamento farmacológico continuado para manter-se 
bem, deve-se cogitar e apresentar a alternativa do tratamento cirúrgico. Nesta indicação, pesam vários fatores tais como idade e condições clínicas, o tipo de droga necessária para mantê-lo assintomático e análise das limitações impostas em relação à qualidade de vida do paciente submetido ao tratamento clínico por longo prazo.

Aspecto importante a ser considerado é que os IBP controlam adequadamente os sintomas ácido-dependentes da DRGE. Porém, não têm efeito adequado no controle de sintomas decorrentes da presença física do refluxo (independentes da acidez do mesmo). VELA et al. ${ }^{(40)}$, avaliando por meio de impedanciopHmetria esofágica a eficiência do omeprazol no controle do RGE, observaram que o uso do fármaco não reduzia significantemente o número de episódios de refluxo, mas sim alterava sua acidez (continua havendo o mesmo número de refluxos, contudo eles tornavamse "não-ácidos"). Portanto, espera-se melhora clínica com o uso da droga, dos sintomas ácido-dependentes, explicando-se a manutenção da queixa de sintomas não-ácido-dependentes.

SHAY et al. ${ }^{(31)}$, estudando pacientes que permanecem sintomáticos apesar do uso de IBP, por meio de impedanciopHmetria, observaram que $28 \%$ deles apresentavam sintomas relacionados com refluxo ácido (detectável por pHmetria convencional). Contudo, $40 \%$ deles apresentavam sintomas relacionados com refluxo não-ácido (detectável pela impedânciopHmetria). Esses autores descrevem que nos $32 \%$ restantes, os sintomas não foram relacionados com refluxo (ácido e não-ácido).

\section{TRATAMENTO CIRÚRGICO}

Por muito tempo preconizou-se que o tratamento cirúrgico da DRGE estaria indicado nos casos de intratabilidade clínica e nas formas complicadas da doença. Com o significativo aumento da eficiência das drogas usadas no tratamento clínico da afecção, pode-se dizer que, atualmente, é menos freqüente a observação de intratabilidade clínica, devendo os pacientes em tal situação ser meticulosamente estudados para conferir a real participação do RGE no desencadeamento dos sintomas. O que se observa na prática é que a maioria dos pacientes tem boa resposta às medidas clínicas usualmente empregadas. Entretanto, parte significativa dos enfermos (cerca de 50\%) tem necessidade de manutenção de tratamento clínico prolongado (comportamental e/ou medicamentoso) para manter-se assintomático ${ }^{(28)}$.

Atualmente, o ponto crucial da indicação do tratamento cirúrgico é a tolerância do paciente ao tratamento clínico prolongado. Alguns deles preferem manter restrições comportamentais e, freqüentemente, o uso de medicamentos, a serem operados; outros preferem o contrário. Cabe ao médico apresentar ao paciente, com imparcialidade, as opções terapêuticas (clínico prolongado e cirúrgico), ponderando vantagens e desvantagens e, compartilhar com o mesmo, a decisão da modalidade de tratamento a ser utilizada.

Estudo baseado em revisão sistemática de literatura ${ }^{(1)}$, comparando a eficácia dos tratamentos clínico e cirúrgico da DRGE crônica, conclui que: "o tratamento cirúrgico é mais eficaz que o tratamento clínico em relação à melhora dos sintomas e cicatrização da esofagite. Contudo, os IBP podem proporcionar melhora dos sintomas comparável ao tratamento cirúrgico, se forem utilizadas doses ajustadas da medicação"; ou seja, aumentase a dose até se conseguir bloqueio adequado da secreção ácida do estômago, avaliada por $\mathrm{pHmetria}$ esofágica prolongada. Vale destacar que, apesar dessa consideração ser válida para o controle das queixas clínicas ácido-dependentes do refluxo, pode não ser para o controle das queixas não-ácido-dependentes.

\section{Indicações}

1. Intolerância ao controle clínico prolongado - considerar que os pacientes em que se observa melhores resultados funcionais do tratamento cirúrgico são os que tiveram boa resposta ao tratamento clínico, mas que se tornaram dependentes do mesmo para se manterem assintomáticos.

2. Formas complicadas da doença (esôfago de Barrett, ulceração, estenose) - apesar da indicação cirúrgica nas formas complicadas da DRGE ser menos polêmica do que nas formas não-complicadas, não se deve indicar a terapêutica cirúrgica simplesmente pela existência de uma das complicações da doença. Esses pacientes devem ser cuidadosamente estudados buscando-se compreender a fisiopatogenia da complicação, indicando-se o tratamento mais adequado para cada caso.

O risco de adenocarcinoma do esôfago é maior dentre os indivíduos com esôfago de Barrett. Contudo, existe grande polêmica quanto à capacidade do tratamento cirúrgico diminuir tal risco. Estudo baseado em revisão sistemática de literatura ${ }^{(9)}$, avaliando o efeito do tratamento cirúrgico do refluxo no risco de adenocarcinoma no esôfago de Barrett, conclui que: "o risco de adenocarcinoma em indivíduos com esôfago de Barrett é pequeno e não é reduzido de modo significante, pelo tratamento cirúrgico do refluxo. Em função disso, o tratamento cirúrgico do refluxo gastroesofágico não deve ser recomendado como medida antineoplásica".

3. Pacientes com manifestações respiratórias da DRGE existe associação freqüente entre a ocorrência de sintomas respiratórios e RGE. Entretanto, a relação de causa e efeito nem sempre é de fácil comprovação. Alguns pacientes com problemas respiratórios primários desenvolvem secundariamente DRGE pelo uso de medicamentos que reduzem o tônus pressórico do esfíncter inferior do esôfago e, por vezes, por deformidades torácicas associadas que favorecem o RGE. Outros enfermos apresentam primariamente RGE e manifestações respiratórias decorrentes do mesmo. Quando ficar bem esclarecida a participação do RGE na determinação e/ou perpetuação dos sintomas respiratórios, a correção cirúrgica do refluxo é bem indicada.

Alguns estudos apontam que mais da metade dos pacientes asmáticos apresenta evidências endoscópicas ou pHmétricas indicativas da DRGE. Contudo, a relação entre causa e efeito entre essas duas condições (asma e DRGE) nem sempre é de fácil entendimento, pois o RGE pode ser causa, mas também pode ser conseqüência da asma e do seu tratamento. Existe controvérsia quanto ao valor da terapia anti-refluxo na asma.

SONTAG et al. ${ }^{(35)}$ compararam os resultados obtidos com as seguintes modalidades de tratamento: fundoplicatura tipo Nissen, 
ranitidina ( $150 \mathrm{mg}$ três vezes ao dia) e alcalinos. Foram analisados os sintomas de asma por meio de pontuação na qual se considerava, além das queixas clínicas, a necessidade de uso de broncodilatadores e corticóides. Os autores referem melhora parcial ou completa em $75 \%$ dos pacientes tratados cirurgicamente, em $9 \%$ dos tratados com ranitidina e $4 \%$ no tratado apenas com alcalinos. O uso de corticóides foi interrompido em $33 \%$ do grupo tratado cirurgicamente, $11 \%$ do grupo tratado com ranitidina e em nenhum caso do grupo tratado com alcalino. Deve-se destacar, entretanto, que as drogas utilizadas no grupo tratado farmacologicamente não são as mais eficientes disponíveis.

Há vários estudos de revisão analisando a alta freqüência de RGE em pacientes com asma e as dificuldades diagnósticas existentes em saber a real participação do refluxo no quadro respiratório. Contudo, as poucas revisões sistemáticas publicadas apresentam resultados conflitantes ${ }^{(7,14,18,33,41)}$

Estudo baseado em revisão sistemática de literatura ${ }^{(15)}$, avaliando a eficácia do tratamento cirúrgico do refluxo no controle da asma, conclui que: "o tratamento cirúrgico do refluxo melhora os sintomas do refluxo e da asma e reduzem a necessidade de medicamentos, mas tem pouco ou nenhum efeito sobre a função pulmonar".

Outra publicação baseada em revisão sistemática de literatura ${ }^{(16)}$, avaliando a eficácia do tratamento cirúrgico do refluxo no controle da asma, conclui que: "em termos gerais, não há melhora significante da asma após o tratamento do refluxo. Contudo, podem haver sub-grupos de pacientes que se beneficiam substancialmente com o tratamento do refluxo; a grande dificuldade é predizer quais são os bons respondedores". Talvez, o desafio atual em relação a essa questão seja identificar o sub-grupo de pacientes com asma e refluxo gastroesofágico, nos quais o tratamento do refluxo possa ser benéfico em relação ao controle da asma.

Considera-se, atualmente, que o acesso videolaparoscópico seja mais vantajoso que o acesso por laparotomia (cirurgia aberta). Entretanto, destaca-se que é de fundamental importância que o tratamento cirúrgico seja realizado por equipes de grande experiência no tratamento cirúrgico do refluxo.

Estudo baseado em revisão sistemática de literatura ${ }^{(5)}$, comparando resultados da videocirurgia com a operação convencional (técnica aberta), conclui que: "a videocirurgia é no mínimo tão segura e eficiente quanto a operação pela técnica aberta e proporciona menor morbidade, menor tempo de permanência hospitalar e recuperação mais rápida".

\section{TRATAMENTO ENDOSCÓPICO}

Várias modalidades de tratamento endoscópico da DRGE (plicatura endoscópica, aplicação de radiofreqüência e injeção de polímeros na transição esôfago-gástrica) têm sido desenvolvidas. Porém, até o presente momento, há apenas estudos avaliando os resultados a curto prazo dessas modalidades terapêuticas. Tais estudos têm evidenciado melhora da queixa de pirose, mas não normalização do refluxo à pHmetria e nem cicatrização da esofagite endoscópica. Algumas complicações (incluindo óbitos) têm sido relatadas. Até o momento, não há evidências favoráveis suficientes que autorizem a utilização de tais modalidades de tratamento na prática clínica assistencial. Os estudos relativos ao tema devem continuar restritos a centros de pesquisa e investigação, até que se produzam resultados mais consistentes.

Estudo baseado em revisão sistemática de literatura( ${ }^{(17)}$, avaliando o tratamento endoscópico da DRGE, conclui que: "apesar de haver resultados favoráveis, não há estudos bem controlados suficientes que justifiquem a aplicação clínica do tratamento endoscópico do refluxo".

\section{CONCLUSÕES}

Com base na revisão ora apresentada, pode-se salientar que: 1. DRGE é a afecção crônica decorrente do fluxo retrógrado de parte do conteúdo gastroduodenal para o esôfago e/ou órgãos adjacentes, acarretando variável espectro de sintomas (esofágicos ou extra-esofágicos), associados ou não a lesões teciduais.

2. As manifestações clínicas consideradas típicas da doença são pirose e regurgitação. Mais recentemente, reconheceuse que outras manifestações clínicas (atípicas) podem ser decorrentes de refluxo gastroesofágico. Considera-se que dor torácica não-coronariana, globus faríngeo, manifestações extra-esofágicas respiratórias e otorrinolaringológicas possam também ser decorrentes de RGE.

3. Pacientes com manifestações atípicas podem não apresentar sintomas típicos do refluxo.

4. A endoscopia digestiva alta e a pHmetria esofágica prolongada são os dois métodos diretamente relacionados com o diagnóstico da DRGE. O primeiro identificando as formas da doença que cursam com esofagite e o segundo caracteriza o sub-grupo RGE patológico sem esofagite.

5. A ausência de esofagite ao estudo endoscópico não exclui o diagnóstico da DRGE.

6. impedanciometria esofágica tem contribuído substancialmente para o melhor conhecimento da DRGE e se desponta, quando associada à pHmetria (impedanciopHmetria esofágica), como o novo padrão-ouro para o diagnóstico do RGE.

7. Os IBP aliviam os sintomas do refluxo e cicatrizam a esofagite mais rapidamente que os $\mathrm{AH}_{2}$.

8. Evidências atuais sugerem que os novos IBP (lansoprazol, rabeprazol e pantoprazol) têm eficiência semelhante ao omeprazol no controle da pirose, na taxa de cicatrização de esofagite e no controle da recidiva.

9. Dentre os sintomas da DRGE há os que podem ser considerados ácido-dependentes, que costumam responder bem ao tratamento com IPB. Contudo, há também sintomas que são mais dependentes da presença física do refluxo que da sua acidez. Esses, que não dependem tanto do teor ácido do refluxo, não são bem controlados pelo uso de IBP.

10. O maior problema do tratamento clínico da DRGE não é apenas controlar os sintomas, mas sim manter os pacientes assintomáticos a longo prazo. Além disso, a eliminação dos sintomas de refluxo não necessariamente assegura a normalização do $\mathrm{pH}$ intraesofágico e intragástrico. Frente ao paciente que necessita de tratamento farmacológico continuado para manter-se bem, deve ser cogitada alternativa do tratamento cirúrgico. Nesta decisão, pesam vários fatores tais como: idade, condições clínicas do 
paciente, tipo de droga necessária para mantê-lo assintomático e análise das limitações impostas em relação à qualidade de vida do paciente submetido ao tratamento clínico prolongado.

11. O tratamento cirúrgico da DRGE, fundamentalmente, é indicado:

- intolerância ao controle clínico prolongado;

- formas complicadas da doença (esôfago de Barrett, ulceração, estenose);

- pacientes com manifestações atípicas que têm as queixas decorrentes de refluxo não-ácido e que portanto, apesar de terem refluxo, não respondem bem ao tratamento com IBP.

12. Várias modalidades de tratamento endoscópico da DRGE têm sido desenvolvidas. Contudo, até o presente momento, não existem evidências favoráveis suficientes que autorizem a utilização do tratamento endoscópico do refluxo na prática clínica assistencial. Os estudos relativos ao tema devem continuar restritos a centros de pesquisa e investigação, até que se produzam resultados mais consistentes.

Nasi A, Moraes-Filho JPP, Cecconello I. Gastroesophageal reflux disease: an overview. Arq Gastroenterol. 2006;43(4):334-41.

ABSTRACT - Background - Gastroesophageal reflux disease, considered one of the most common digestive diseases in western countries, has been very much studied. The great number of publications in medical literature based upon this issue must be pointed out. However, some of its aspects remain controversial. Aims - To emphasize important topics of the disease and to present a review on the theme. The following topics were reviewed: definition, prevalence, clinical complaints, indications and limitations of the major diagnostic methods and medical, surgical and endoscopic treatments. Material and methods - We searched, by internet: selected review articles, consensus, guidelines and systematic reviews with meta-analysis, published in the last 5 years (from 2000 to 2005), in the following data bases: Cochrane Central Register of Controlled Trials (The Cochrane Library) and PubMed. Among lots of papers which were found, we selected nine systematic reviews with meta-analysis, five review articles, five guidelines and four consensus, that dealt with the topics we had decided to review. It must be pointed out that, as it has been observed in our bibliographical references, not only the publications found were taken into account; other relevant ones (some of them published previously to the analyzed time period) were considered in the composition of this present paper. Conclusions - Among the 12 conclusions presented, two have to be emphasized: 1. the esophageal multichannel intraluminal impedance has largely contributed to the better knowledge of the gastroesophageal reflux disease and it suggests, when associated with pHmetry (esophageal impedance-pHmetry), as a new gold standard to the gastroesophageal reflux diagnosis and 2: among the gastroesophageal reflux disease symptoms some of them may be considered acid dependents and can usually get good responses to the proton pump inhibitors. Nevertheless, there are also some symptoms which are more dependent on the physical presence of the reflux rather than its acidity. Such symptoms, which do not depend so much on the acidity of the reflux, are not well controlled by the proton pump inhibitors.

HEADINGS - Gastroesophageal reflux. Esophagitis. Esophageal motility disorders. 


\section{REFERÊNCIAS BIBLIOGRÁFICAS}

1. Allgood PC, Bachmann M. Medical or surgical treatment for chronic gastroesophageal reflux? A systematic review of published evidence of effectiveness. Eur J Surg. 2000;166:713-21.

2. American Gastroenterological Association. Technical review on the clinical use of esophageal manometry. Gastroenterology. 2005;128:209-24.

3. Bardhan KD. Reflux rising - a burning issue! A personal overview of treatment. Res Clin Forums. 1998;20:27-32.

4. Caro JJ, Salas M, Ward A. Healing and relapses rates in gastroesophageal reflux disease treated with the newer proton-pump inhibitors lansoprazole, rabeprazole, and pantoprazole compared with omeprazole, ranitidine and placebo: evidence from randomized clinical trials. Clin Ther. 2001;23:998-1017.

5. Catarci M, Gentileschi P, Papi C, Carrara A, Marrese R, Gaspari AL, Grassi GB. Evidence-based appraisal of antireflux fundoplication. Ann Surg. 2004;239:325-37.

6. Chiba N, De Gara CJ, Wilkinson JM, Hunt RH. Speed of healing and symptom relief in grade II to IV gastroesophageal reflux disease: a meta-analysis. Gastroenterology. 1997;112:1798-810.

7. Choy D, Leung R. Gastro-oesophageal reflux and asthma. Respiratory. 1997;2:163-8.

8. Christopher G, Streets MRCS, DeMeester TR. Ambulatory 24-hour esophageal pH monitoring: why, when and what to do? J Clin Gastroenterol. 2003;37:14-22.

9. Corey KE, Schmitz SM, Shaheen NJ. Does a surgical antireflux procedure decrease the incidence of esophageal adenocarcinoma in Barrett's esophagus? A meta-analysis. Am J Gastroenterol. 2003;98:2341-2.

10. De Vault KR, Castell DO. Updated guidelines for the treatment of gatroesophageal reflux disease. Am J Gastroenterol. 2005;100:190-200

11. Dent J, Brun J, Fendrick AM, Fennerty MB, Janssens J, Kahrilas PJ, Lauritsen K, Reynolds JC, Shaw M, Talley NJ. An evidence-based appraisal of reflux disease management. The Genval Workshop Report. Gut. 1999;44(Suppl 2):S1-S16.

12. DeVault KR, Castell DO. Updated guidelines for the diagnosis and treatment of gastroesophageal reflux disease. The Practice Parameters Committee of the American College of Gastroenterology. Am J Gastroenterol. 1999;94:1434-42.

13. Donnellan C, Sharma N, Preston C, Moayyedi P. Medical treatments for the maintenance therapy of reflux oesophagitis and endoscopic negative reflux disease (Cochrane Review). In: The Cochrane Library, Issue 2. Oxford: Update Software; 2005.

14. Field SK, Gelfand GA, McFadden SD. The effects of antireflux surgery on asthmatics with gastroesophageal reflux. Chest. 1999;116:766-74.

15. Field SK, Sutherland LR. Does medical therapy improve asthma in asthmatics with gastroesophageal reflux: a critical review of the literature. Chest. 1998;114:275-83.

16. Gibson PG, Henry PL, Coughlan JL. Gastro-oesophageal reflux treatment for asthma in adults and children (Cochrane Review). In: The Cochrane Library, Issue 2. Oxford: Update Software; 2005

17. Johnson DA. Endoscopic therapy for gastroesophageal reflux disease: a systematic review. Minerva Gastroenterol Dietol. 2004;50:239-51.

18. Karilas PJ. Gastroesophageal reflux disease. JAMA. 1996;276:983-8.

19. Katelaris P, Holloway R, Talley N, Gotley D, Williams S, Dent J, Digestive Health Foundation of the Gastroenterological Society of Australia. Gastro-oesophageal reflux disease in adults: guidelines for clinicians. J Gastroenterol Hepatol. 2002;17:825-33.

20. Katz PO. Treatment of gastroesophageal reflux disease: use of algorithms to aid in management. Am J Gastroenterol. 1999;94:s3-s10.

21. Klinkenberg-Knol EC, Festen HP, Jansen JB, Lamers CB, Nelis F, Snel P, Luckers A, Dekkers CP, Havu N, Meuwissen SG. Long-term treatment with omeprazole for refractory reflux esophagitis: efficacy and safety. Ann Intern Med. 1994:121:161-7.

22. Moraes-Filho JPP, Cecconello I, Gama-Rodrigues J, Paula-Castro L, Henry MA, Meneghelli UG, Quigley E. Brazilian Consensus on Gastroesophageal Reflux Disease: proposals for assessment, classification, and management. Am J Gastroenterol. 2002;97:241-8.

23. Moraes-Filho JPP, Chinzon D, Eisig JN. Prevalence of heartburn and gastroesophageal reflux disease in the urban Brazilian population. Gastroenterology. 2003;124;4 (Suppl 1):A-166.
24. Moss SF, Arnold R, Tytgat GN, Spechler SJ, Delle-Fave G, Rosin D, Jensen RT, Modlin IM. Consensus Statement for Management of Gastroesophageal Reflux Disease: result of workshop meeting at Yale University School of Medicine, Department of Surgery, November 16 and 17, 1997. J Clin Gastroenterol. 1998;27:6-12.

25. Nasi A, Moraes-Filho JPP, Zilberstein B, Cecconello I, Gama-Rodrigues JJ, Pinotti HW. Gastroesophageal reflux disease: clinical, endoscopic and intraluminal esophageal pH monitoring evaluation. Dis Esophagus. 2001;14:41-9.

26. Numans ME, Lau J, de Wit NJ, Bonis PA. Short-term treatment with proton-pump inhibitors as a test for gastroesophageal reflux disease: a meta-analysis of diagnostic test characteristics. Ann Intern Med. 2004;140:518-27.

27. Orlando RC. Why is the high-grade inhibition of gastric acid secretion afforded by proton pump inhibitors often required for healing of reflux esophagitis? Am J Gastroenterol. 1996;91:1692-6.

28. SAGES guidelines. Guidelines for surgical treatment of gastroesophageal reflux disease. Surg Endosc. 1998;12:186-8.

29. Sandmark S, Carlsson R, Lundell L. Omeprazole or ranitidine in the treatment of reflux esophagitis. Scand J Gastroenterol. 1988;23:625-32.

30. Shay S, Tutuian R, Sifrim D, Vela M, Wise J, Balaji N, Zhang X, Adhami T, Murray $\mathrm{J}$, Peters J, Castell D. Twenty-four hour ambulatory simultaneous impedance and $\mathrm{pH}$ monitoring: a multicenter report of normal values from 60 healthy volunteers. Am J Gastroenterol. 2004;99:1037-43.

31. Shay S, Tutuian R, Sifrim D, Vela M, et al. Twenty-four hour impedance and $\mathrm{pH}-$ monitoring in the evaluation of GERD patients with persistent symptoms despite BID proton pump inhibitors: a multicenter study [abstract]. DDW 2004. Gastroenterology. 2004;126 Suppl 2:A324

32. Sifrim D, Castell DO, Dent J, Kahrilas PJ. Gastro-oesophageal reflux monitoring: review and consensus report on detection and definitions of acid, non-acid, and gas reflux. Gut 2004;53:1024-31.

33. Simpson WG. Gastroesophageal reflux and asthma. Arch Intern Med. 1995; 155:798-803

34. Sonnenberg A, El-Serag HB. Clinical epidemiology and natural history of gastroesophageal reflux disease. Yale J Biol Med. 1999;72:81-9.

35. Sontag SJ, O'Connell S, Khandelwal S, Miller T, Nemchausky B, Schnell TG, Serlovsky R. Most asthmatics have gastroesophageal reflux with or without bronchodilator therapy. Gastroenterology. 1990;99:613-20.

36. Torquati A, Lutfi R, et al. Laparoscopic fundoplication: is it worthwhile in patients with persistent GERD symptoms despite PPI therapy? [abstract] DDW 2004. 2004;126 Suppl 2:A330.

37. Tutuian R, Castell DO. Clarification of the esophageal function defect in patients with manometric ineffective esophageal motility: studies using combined impedancemanometry. Clin Gastroenterol Hepatol. 2004;2:230-6

38. Tutuian R, Castell DO. Use of multichannel intraluminal impedance to document proximal esophageal and pharyngeal nonacidic reflux episodes. Am J Med. 2003;115(Suppl $3 \mathrm{~A}): 119 \mathrm{~s}-23 \mathrm{~s}$.

39. Van Pinxteren B, Numanas ME, Bonis PA, Lau J. Short-term treatment with protonpump inhibitors, $\mathrm{H}_{2}$-receptor antagonists and prokinetics for gastro-oesophageal reflux disease-like symptoms and endoscopy negative reflux disease. (Cochrane Review). In: The Cochrane Library, Issue 2, 2005. Oxford: Update Software.

40. Vela MF, Camacho-Lobato L, Srinivasan R, Tutuian R, Katz PO, Castell DO. Simultaneous intraesophageal impedance and $\mathrm{pH}$ measurement of acid and nonacid gastroesophageal reflux: effect of omeprazole. Gastroenterology. 2001;120:1599-606.

41. Winter DC, Brennan NJ, O'Sullivan G. Reflux induced respiratory disorders. J Irish Coll Phys Surg. 1997;26:202-10. 